\title{
H I Tidal Tails, Bridges and Clouds
}

\author{
Bärbel S. Koribalski \\ Australia Telescope National Facility, CSIRO, Australia
}

\begin{abstract}
There is plenty of intergalactic H I gas without any obvious stellar content ranging from (1) extended gas envelopes around some normal and peculiar galaxies, (2) tidal tails/bridges in interacting or merging galaxy systems, (3) large-scale rings around early type galaxies, and (4) detached clouds at varying distances from associated galaxies, but there are few or no isolated $\mathrm{H}$ I clouds.

The HIPASS Bright Galaxy Catalog, which covers the whole southern sky, contains only one definite extragalactic $\mathrm{H}$ I cloud which is located close to the galaxy NGC 2442 whereas it is sensitive to isolated H I clouds with $M_{\mathrm{HI}}>10^{6} \times D^{2} \mathrm{M}_{\odot}$. The space density of $\mathrm{H}$ I clouds is therefore about $1 / 1000$ th that of galaxies with the same $M_{\mathrm{HI}}$.
\end{abstract}

\section{Introduction}

The best resource to marvel at the peculiar $\mathrm{H}$ I distribution in interacting galaxy pairs/groups and some individual galaxies is the "H I Rogues Gallery" compiled by Hibbard et al. (2001; see www.nrao.edu/astrores/HIrogues). Here we will concentrate on extragalactic $\mathrm{H}$ I gas that has no obvious stellar counterpart. This gas is found mostly in the outskirts of galaxies, in the form of extended envelopes, tidal tails, bridges, rings and detached clouds. Prominent nearby examples include the Magellanic Stream (Putman et al. 2003) and the extended H I bridges in the M 81 group (Yun et al. 1994, Makarova et al. 2002). The questions we want to address are: how much intergalactic $\mathrm{H}$ I gas is there, where is it located and what is its fate? In particular, how much gas is ejected into the intergalactic medium by tidal forces? We will look at the amount and location of the $\mathrm{H}$ I gas surrounding peculiar/interacting galaxies.

Hibbard \& Mihos (1995) used numerical simulations to study what happens to tidal debris over a long period of time. They found that a large fraction of the ejected H I gas will fall back onto the parent galaxy. The closest material falls back fastest, while more distant debris return ever more slowly to accrete at larger distances from the galaxy centre. That leaves plenty of time for the slowly returning material as well as any escaped gas to form bound entities and potentially stars. Some of these star forming clumps will build new galaxies, such as tidal dwarf galaxies (Barnes \& Hernquist 1992; Duc et al. 2000, Braine et al. 2001). But, how long can these young, recycled objects survive in an interacting galaxy environment? 


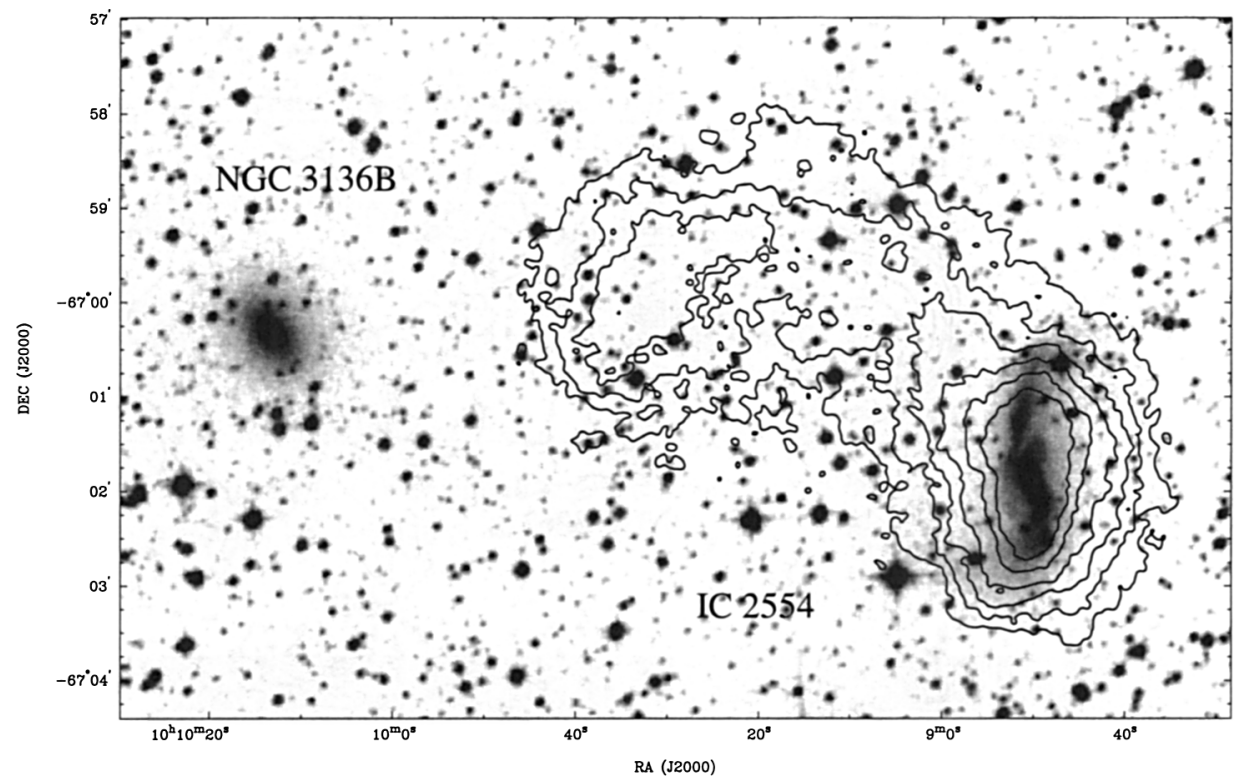

Figure 1. HI distribution (contours) towards the spiral galaxy IC 2554 overlaid onto a DSS II R-band image. The large, one-sided H I plume possibly results from tidal interaction with the massive, elliptical galaxy NGC 3136B. For details see Koribalski et al. (2003).

Some galaxies have very large $\mathrm{H}$ I envelopes extending way beyond their stellar distribution (Broeils \& van Woerden 1994, Salpeter \& Hoffman 1996; Broeils $\&$ Rhee 1997). The nature of the extended gas distribution can be very different and ranges from smooth disks to chaotic extensions. Because of its large extent, the outer neutral hydrogen disk is much more affected by tidal interactions than the stellar disk and therefore an excellent tracer of these. Collisions of galaxies with extended $\mathrm{H}$ I envelopes lead to large $\mathrm{H}$ I extensions, tails and bridges which can be found out to large distances from the galaxy centre. Recycling and reaccretion of these distant debris are increasingly important for the evolution of these systems. We will look at galaxies with (1) extended $\mathrm{H}$ I envelopes, (2) tidal H I tails/plumes, (3) large, possibly accreted H I rings, (4) detached H I clouds, and examine their extent, mass, and structure. In each category we can only give a few examples. A thorough compilation of such systems, including a set of standard properties for each, would be most useful for many observational and numerical studies.

\section{Galaxies with extended $H_{I}$ envelopes}

While the average $\mathrm{H}$ I to optical extent of galaxies is less than two, a few galaxies have very extended $\mathrm{HI}$ distributions. We give a few examples (see Table 1) and compare the measured $\mathrm{H}$ I extent to the optical Holmberg radius, $R_{\mathrm{Ho}}$. Ideally we would like to quote standard $\mathrm{H}$ I radii at certain $\mathrm{H}$ I column or surface densities similar to the standard optical radii at certain magnitudes. 


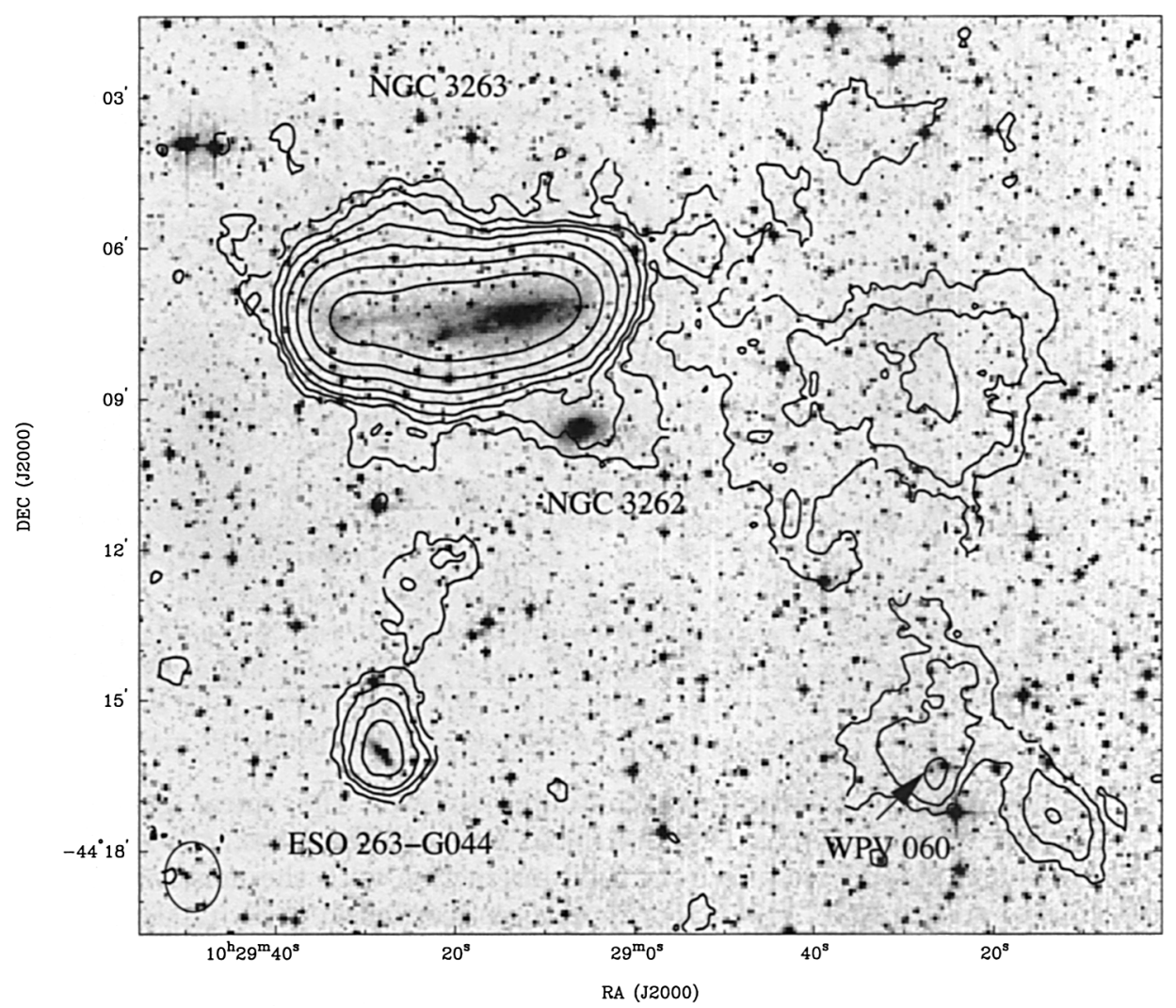

Figure 2. Hi distribution (contours) towards the peculiar galaxy NGC 3263 overlaid onto a DSS II B-band image. The spectacular H I plume to the west/south-west of NGC 3263/2 extends over $\sim 175 \mathrm{kpc}$ $\times 200 \mathrm{kpc}$. The dwarf galaxy WPV 060 coincides with a peak in the $\mathrm{H} \mathrm{I}$ plume and is marked with an arrow. For details see English, Koribalski \& Freeman (these proceedings).

Note that while the H I masses of NGC 2915, DDO 154 and ESO215-G?009 are similar, their $\mathrm{H}$ I mass to light ratios are very different with $M_{\mathrm{HI}} / L_{\mathrm{B}}=$ $2.6,8$, and $\sim 21$, respectively. The $\mathrm{H}$ I distribution of the Magellanic irregular NGC 4449, also known as the "Jekyll \& Hyde" galaxy is enormous and peculiar. Hunter et al. (1998) find $\sim 10^{9} \mathrm{M}_{\odot}$ in the $\mathrm{H}$ I streamers alone (> $\left.35 \mathrm{kpc}\right)$.

\section{Galaxies with $\mathrm{H}$ I tails/plumes}

The galaxy systems listed in Table 2 have enormous, one-sided tidal tails and are typically mergers. These are quite distinct from the more diffuse, one-sided H I plumes/clouds observed towards, e.g., the spiral galaxies IC 2554 (see Fig. 1) and NGC 3263 (see Fig. 2). The prominent plume emerging to the east of the peculiar galaxy IC $2554(D=16 \mathrm{Mpc})$ is possibly caused by interaction with the massive elliptical galaxy NGC 3136B (Koribalski et al. 2003). It has 
Table 1. Examples of galaxies with an extended $\mathrm{H}$ I distribution

\begin{tabular}{lcccccc}
\hline Name & type & $\begin{array}{c}D \\
{[\mathrm{Mpc}]}\end{array}$ & $\begin{array}{c}\text { H I extent } \\
{[\mathrm{kpc}]}\end{array}$ & $\times R_{\mathrm{Ho}}$ & $\begin{array}{c}M_{\mathrm{HI}} \\
{\left[10^{9} \mathrm{M}_{\odot}\right]}\end{array}$ & Refs. \\
\hline NGC 2915 & BCD & 2.7 & 16 & 5 & 0.25 & $(1)$ \\
DDO 154 & IBm & 3.2 & 8.4 & 5 & 0.25 & $(2)$ \\
ESO215-G?009 & dIrr & 4.2 & 15 & 5 & 0.5 & $(3)$ \\
NGC 4449 & IBm & 3.4 & 66 & 6 & 2 & $(4)$ \\
Circinus & SAb & 4.2 & $>73$ & $>4$ & 8 & $(5)$ \\
M 83 & SABc & 4.5 & 78 & 4 & 8 & $(6)$ \\
\hline
\end{tabular}

(1) Meurer et al. 1996, (2) Carignan \& Purton 1998, (3) Warren, Jerjen, Koribalski, these proceedings, (4) Hunter et al. 1998, (5) Jones et al. 1999, (6) Park et al. 2001.

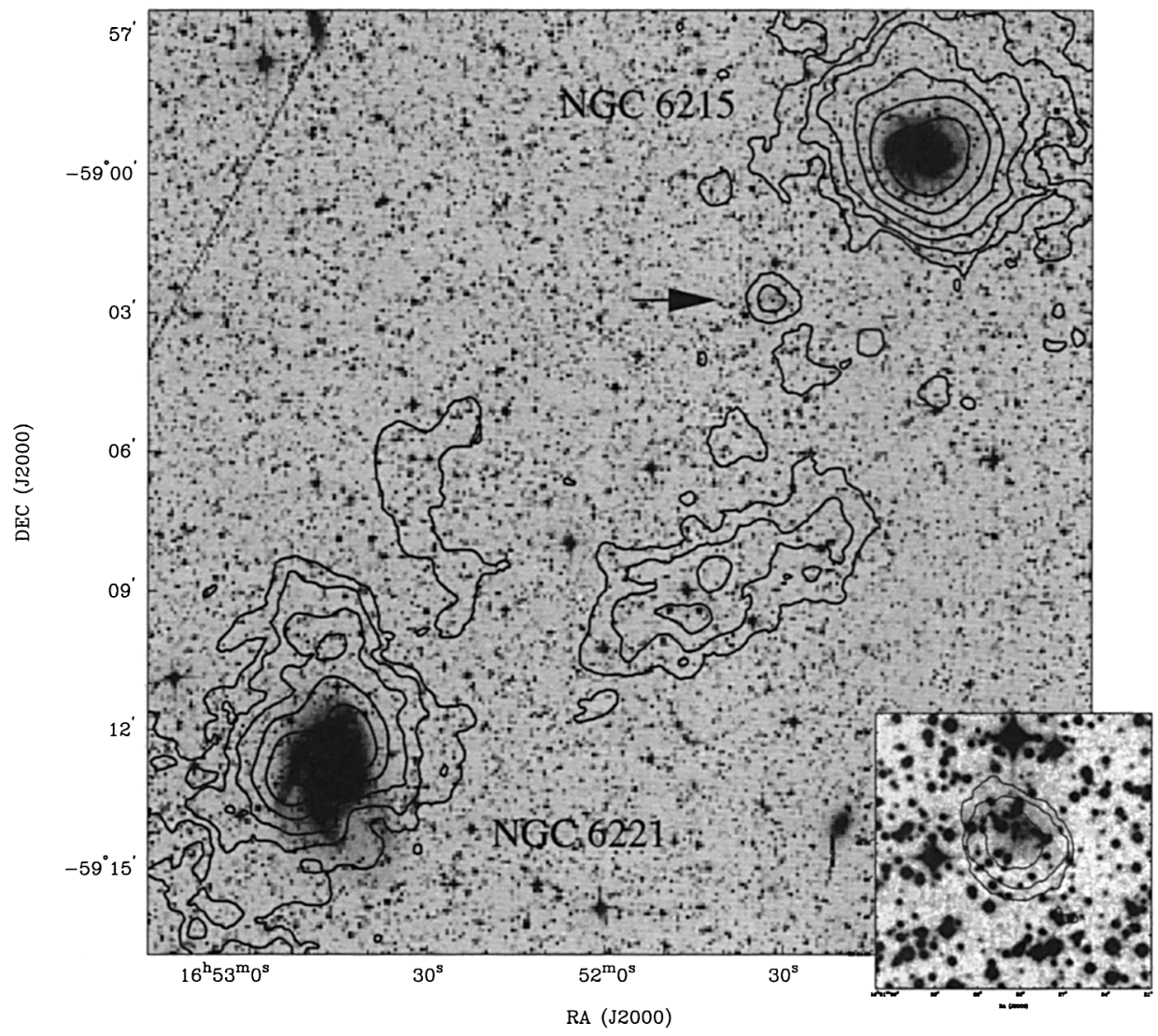

Figure 3. H I distribution (contours) towards the interacting spiral galaxies NGC 6221 and NGC 6215 overlaid onto a DSS II B-band image. For details see Koribalski \& Dickey (2004). 
Table 2. Examples of galaxy pairs with long, one-sided H I tails

\begin{tabular}{lcccc}
\hline Name & $\begin{array}{c}D \\
{[\mathrm{Mpc}]}\end{array}$ & $\begin{array}{c}\mathrm{H} \text { I tail } \\
{[\mathrm{kpc}]}\end{array}$ & $\begin{array}{c}M_{\mathrm{HI}} \\
{\left[10^{9} \mathrm{M}_{\odot}\right]}\end{array}$ & Refs. \\
\hline Arp 85 (M 51, NGC 5194/5) & 9.6 & 90 & 0.5 & $(1)$ \\
Arp 143 (NGC 2444/5) & 9.6 & 90 & 0.5 & $(2)$ \\
Arp 270 (NGC 3395/6) & 21.7 & 70 & 0.5 & $(3)$ \\
Arp 215 (NGC 2782) & 34 & 54 & 1.4 & $(4)$ \\
Arp 299 (NGC 3690 + IC 694) & 48 & 180 & 3.3 & $(5)$ \\
\hline
\end{tabular}

(1) Rots et al. 1990, (2) Appleton et al. 1987, (3) Clemens et al. 1999, (4) Smith 994, Jogee et al. 1998, (5) Hibbard \& Yun 1999.

an extent of $\sim 30 \mathrm{kpc}$ and contains about a third of the total H I mass of the system $\left(2 \times 10^{9} \mathrm{M}_{\odot}\right)$. The NGC 3256 galaxy group $(D=37.6 \mathrm{Mpc})$ features a spectacular $\mathrm{H}$ I cloud of size $\sim 175 \mathrm{kpc} \times 100 \mathrm{kpc}$ with an $\mathrm{H}$ I mass of $10^{9} \mathrm{M}_{\odot}$ (English, Koribalski \& Freeman; these proceedings). It lies just west of the $\mathrm{H} \mathrm{I}$ rich galaxy NGC 3263 which in itself is most peculiar (Koribalski et al. 2004, in prep.). The dwarf galaxy WPV 060 which appears to be associated with an H I peak towards the south-western end of the cloud, could be a young galaxy formed out of the far-reaching tidal debris.

\section{H I rings}

In Table 3 we list a few examples of $\mathrm{E} / \mathrm{S} 0$ galaxies surrounded by large $\mathrm{H}$ I rings. Duc \& Mirabel (1998) suggest that the H I ring in the NGC 5291 system, which appears to be a nursery of recycled dwarf galaxies, was accreted from a gas-rich galaxy in the cluster.

Table 3. Examples of galaxies surrounded by large $\mathrm{H}$ i rings

\begin{tabular}{lccccc}
\hline Name & type & $\begin{array}{c}D \\
{[\mathrm{Mpc}]}\end{array}$ & $\begin{array}{c}\text { H I ring } \\
{[\mathrm{kpc}]}\end{array}$ & $\begin{array}{c}M_{\mathrm{HI}} \\
{\left[10^{9} \mathrm{M}_{\odot}\right]}\end{array}$ & Refs. \\
\hline NGC 5291 & E & 58 & 170 & 50 & $(1)$ \\
Leo Ring (M 96 group) & E/S0 & 10 & 200 & 1 & $(2)$ \\
NGC 1533 & S0 & 21 & 70 & 7 & $(3)$ \\
NGC 2292/3 & S0 & 27 & 30 & 2 & $(4)$ \\
\hline
\end{tabular}

(1) Malphrus et al. 1997, (2) Schneider et al. 1989, (3) Ryan-Weber et al., these proceedings; Ryan-Weber et al. 2004, (4) Barnes 1999.

\section{Detached H I clouds}

H I clouds are seen in many tidally interacting galaxy groups such as NGC 697 (van Moorsel 1988), the Grus Quartet (Koribalski et al., in prep.), and the NGC $6221 / 15$ group $(D=18 \mathrm{Mpc}$; Koribalski \& Dickey 2004). These may constitute the gaseous material ejected furthest from the host galaxy and now disconnected from gas that has already returned. The two-stranded $\mathrm{H}$ I bridge between the galaxies NGC 6221 and NGC 6215 (Fig. 3) spans over a projected distance of nearly $100 \mathrm{kpc}$. It has an H I mass of at least $1.4 \times 10^{8} \mathrm{M}_{\odot}$. Note 
that Fig. 3 displays only $\mathrm{H}$ I within the bridge velocity range. The NGC 6221/15 group also contains three H I-rich dwarf galaxies, only one of which (Dwarf 3) is shown here, marked with an arrow. The inset at the bottom right shows a close-up of Dwarf 3. Oosterloo et al. (these proceedings) found several extended $\mathrm{H}$ I clouds more than $100 \mathrm{kpc}$ from the elliptical galaxy NGC $1490(D=70$ Mpc) with a total $M_{\mathrm{HI}}$ of almost $10^{10} \mathrm{M}_{\odot}$. H I $1225+01$, better known as "the Virgo Cloud" ( $D=20 \mathrm{Mpc}$ ) has an H I extent over $100 \mathrm{kpc}$ and an $\mathrm{HI}$ mass of $5 \times 10^{9} \mathrm{M}_{\odot}$ (Giovanelli \& Haynes 1989). Only the NE component of the $\mathrm{HI}$ distribution has an optical counterpart (Chengalur et al. 1995).

The only intergalactic $\mathrm{H}$ I gas cloud detected in the HIPASS Bright Galaxy Catalog (BGC; Koribalski et al. 2004) lies at a projected separation of 250 $\mathrm{kpc}$ from the galaxy NGC $2442(D=15.5 \mathrm{Mpc})$ which is part of a loose group of galaxies. The cloud, HIPASS J0731-69, for which no optical counterpart has been detected, has an $\mathrm{H}$ I mass of $10^{9} \mathrm{M}_{\odot}$ (Ryder et al. 2001; Ryder \& Koribalski, these proceedings).

\section{References}

Appleton, P.N., Ghigo, F.D., van Gorkom, J.H., et al. 1987, Nature, 330, 140

Barnes, J.E., \& Hernquist, L. 1992, Nature, 360, 715

Barnes, D.G. 1999, PASA, 16, 77

Braine, J., Duc, P.-A., Lisenfeld, U., et al. 2001, A\&A, 378, 51

Broeils, A.H., \& van Woerden, H. 1994, A\&AS, 107, 129

Broeils, A.H., \& Rhee, M.-H. 1997, A\&A, 324, 877

Carignan, C., \& Purton, C. 1998, ApJ, 506, 125

Chengalur, J.N., Giovanelli, R., Haynes, M.P. 1995, AJ, 109, 2415

Clemens, M.S., Baxter, K.M., Alexander, P., Green, D.A. 1999, MNRAS, 308, 364

Duc, P.-A., \& Mirabel, I.F. 1998, A\&A, 333, 813

Duc, P.-A., Brinks, E., Springel, V., Pichardo, B., Weilbacher, P., Mirabel, I.F. 2000, AJ, 120, 1238

Giovanelli, R., \& Haynes, M.P. 1989, ApJ, 346, L5

Koribalski, B.S., Gordon, S., Jones, K. 2003, MNRAS, 339, 1203

Koribalski, B.S., \& Dickey, J.M. 2004, MNRAS, in press

Koribalski, B.S., et al. 2004, in press

Hibbard, J.E., \& Mihos, J.C. 1995, AJ, 110, 140

Hibbard, J.E., \& Yun, M.S. 1999, AJ, 118, 162

Hibbard, J.E., van Gorkom, J.H., Rupen, M.P., Schiminovich, D. 2001, in "Gas \& Galaxy Evolution", ASP Conf. Series, Vol. 240, eds. J.E. Hibbard, M.P. Rupen, \& J.H. van Gorkom, p. 659

Hunter, D.A., Wilcots, E.M., van Woerden, H., Gallagher, J.S., Kohle, S. 1998, ApJ, 495, L47

Jogee, S., Kenney, J.D.P., Smith, B. 1998, ApJ, 494, L185 
Jones, K.L., Koribalski, B.S., Elmouttie, M., Haynes, R.F. 1999, MNRAS, 302, 649

Malphrus, B.K., Simpson C.E., Gottesman, S.T., Hawarden, T.G. 1997, AJ, 114,1427

Makarova, L.N., Grebel, E.K., Karachentsev, et al. 2002, A\&A, 396, 473

Meurer, G.R., Carignan, C., Beaulieu, S.F., Freeman, K.C. 1996, AJ, 111, 1551

Park, O.-K., Kalnajs, A. Freeman, K.C., Koribalski, B., Staveley-Smith, L. 2001, in "Galaxy Disks and Disk Galaxies", ASP Conf. Series, Vol. 230, eds. J.G. Funes, E. \& M. Corsini, p. 109

Putman, M.E., Staveley-Smith, L., Freeman, K.C., Gibson, B.K., Barnes, D.G. 2003, ApJ, 586, 170

Rots, A.H., Bosma, A., van der Hulst, J.M., Athanassoula, E., Crane, P.C. 1990, AJ, 100,387

Ryan-Weber, E., Meurer, G.R., et al. 2004, AJ, in press

Ryder, S.D., Koribalski, B.S., Staveley-Smith, L., Kilborn, V.A. et al. 2001, ApJ, 555, 232

Salpeter, E.E., Hoffman, G.L. 1996, ApJ, 465, 595

Schneider, S.E. 1989, AJ, 97, 666

Smith, B.J. 1994, AJ, 107, 1695

van Moorsel, G.A. 1988, A\&A, 202, 59

Yun, M., Ho, P., Lo, K. 1994, Nature, 372, 530 